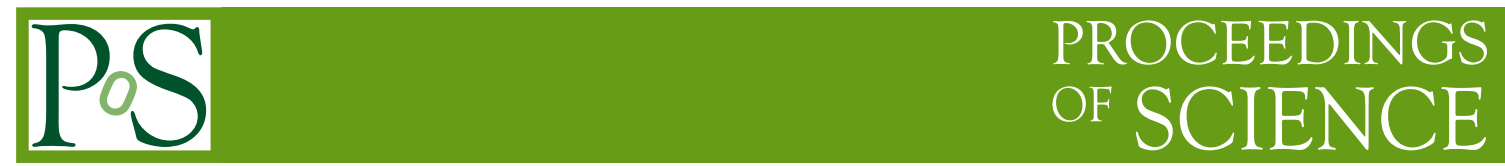

\title{
NNLO corrections to charmless hadronic B decays
}

\author{
Guido Bell*i \\ Institut für Theoretische Teilchenphysik, Universität Karlsruhe, 76128 Karlsruhe, Germany \\ E-mail: belleparticle.uni-karlsruhe.de
}

We report on the status of the perturbative calculation in the QCD factorization framework to charmless hadronic $B$ meson decays and present a compilation of available NNLO results.

International Workshop on Effective Field Theories: from the pion to the upsilon

February 2-6 2009

Valencia, Spain

\footnotetext{
* Speaker.

$\dagger$ I would like to thank Volker Pilipp for a fruitful collaboration on part of the subjects presented in this article and the organizers of EFT09 for creating a pleasant and stimulating workshop atmosphere. This work was supported by the DFG Sonderforschungsbereich/Transregio 9.
} 


\section{Introduction}

$B$ meson decays into a pair of light mesons are mediated by rare flavour-changing $b \rightarrow u$ ("tree") or loop-induced $b \rightarrow d, s$ ("penguin") quark transitions. The plethora of hadronic twobody final states, consisting of e.g. $\pi, \rho, K, K^{*}, \eta$ or $\phi$ mesons, opens a particularly rich laboratory for testing the CKM paradigm of flavour mixing and $\mathrm{CP}$ violation.

In order to exploit the wealth of data that has been collected at the $B$ physics experiments, a quantitative control of the strong-interaction effects is essential. QCD factorization (QCDF) [1] is a systematic framework to compute the hadronic matrix elements from first principles. It is based on the statement that the matrix elements of the operators in the weak effective Hamiltonian factorize in the heavy quark limit $m_{b} \gg \Lambda_{\mathrm{QCD}}$ according to

$$
\begin{aligned}
\left\langle M_{1} M_{2}\left|Q_{i}\right| \bar{B}\right\rangle \simeq & F^{B M_{1}}(0) f_{M_{2}} \int d u T_{i}^{I}(u) \phi_{M_{2}}(u) \\
& +\hat{f}_{B} f_{M_{1}} f_{M_{2}} \int d \omega d v d u T_{i}^{I I}(\omega, v, u) \phi_{B}(\omega) \phi_{M_{1}}(v) \phi_{M_{2}}(u),
\end{aligned}
$$

where the non-perturbative effects are confined to some process-independent hadronic parameters such as decay constants $f_{M}$, light-cone distribution amplitudes $\phi_{M}$ and a heavy-to-light form factor $F^{B M}(0)$ at maximum recoil. The short-distance hard-scattering kernels $T_{i}^{I, I I}$, on the other hand, are perturbatively calculable and currently being worked out to next-to-next-to-leading order (NNLO), i.e. at $\mathscr{O}\left(\alpha_{s}^{2}\right)[2,3,4,5]$. Here we report on the status of the perturbative calculation, which we divide into two parts: vertex corrections $\left(T_{i}^{I}\right)$ and spectator scattering $\left(T_{i}^{I I}\right)$.

\section{Spectator scattering}

We start with the class of short-distance interactions that involves the spectator quark of the decaying $B$ meson. Technically, the calculation of the kernels $T_{i}^{I I}$ amounts at the considered order $\alpha_{s}^{2}$ to a 1-loop calculation with six external legs, cf. the left diagram of Figure 1. The calculation is complicated by the fact that the interactions between the soft spectator and the energetic (collinear) particles in the final state induce a new (virtual) degree of freedom. These so-called hard-collinear or jet modes describe configurations of energetic massless particles with virtualities $\mu_{h c}^{2} \simeq m_{b} \Lambda_{\mathrm{QCD}}$, which is in between the hard scale $\mu_{h} \simeq m_{b}$ and the hadronic scale $\Lambda_{\mathrm{QCD}}$.

The decomposition in (1.1) relies on a perturbative treatment of these hard-collinear modes (it is usually assumed that $\mu_{h c} \simeq 1.5 \mathrm{GeV}$ ). As the kernels $T_{i}^{I I}$ contain the effects from two shortdistance modes with different virtualities, the calculation becomes most transparent when it is

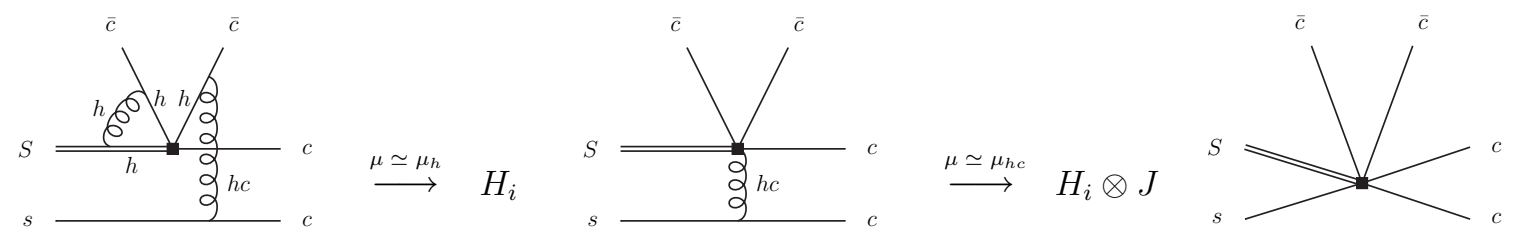

Figure 1: Two-step matching procedure $\mathrm{QCD} \rightarrow \mathrm{SCET}_{\mathrm{I}}(\mathrm{hc}, \mathrm{c}, \mathrm{s}) \rightarrow \mathrm{SCET}_{\mathrm{II}}(\mathrm{c}, \mathrm{s})$ of the spectator scattering contribution. $S(s)$ denotes a soft heavy (light) quark, $c(\bar{c})$ a collinear quark in the direction of the meson $M_{1}$ $\left(M_{2}\right)$ and $h(h c)$ refers to virtual hard (hard-collinear) modes. 
organized as a two-step matching calculation between QCD and soft-collinear effective theory (SCET) [6], where the perturbative degrees of freedom are subsequently integrated out.

The matching calculation is illustrated in Figure 1: QCD is first matched at $\mu \simeq \mu_{h}$ onto an effective theory called $\operatorname{SCET}_{\mathrm{I}}(\mathrm{hc}, \mathrm{c}, \mathrm{s})$ and then at a lower scale $\mu \simeq \mu_{h c}$ onto $\operatorname{SCET}_{\mathrm{II}}(\mathrm{c}, \mathrm{s})$. The respective matching coefficients $H_{i}(J)$ encode the effects of the hard (hard-collinear) modes, while the hadronic matrix element of the remnant non-local operator in $\operatorname{SCET}_{I I}(\mathrm{c}, \mathrm{s})$ yields the factorized light-cone distribution amplitudes $\phi_{M}$ of the three mesons. The hard-scattering kernels $T_{i}^{I I}$ finally follow from a convolution of the perturbative coefficient functions

$$
T_{i}^{I I}(\omega, v, u)=\int d z J(\omega, v, z) H_{i}(z, u)
$$

From Figure 1 it is evident that the spectator scattering mechanism requires at least one perturbative gluon exchange with $J=\mathscr{O}\left(\alpha_{s}\right)$ and $H_{i}=\mathscr{O}(1)$ (at tree level). It has been pointed out in [7] that the same jet function $J$ also enters the factorization formula for heavy-to-light form factors [8]. As the $\mathscr{O}\left(\alpha_{s}^{2}\right)$ corrections to $J$ have already been worked out in this context [9], the NNLO calculation of the kernels $T_{i}^{I I}$ reduces to the computation of the $\mathscr{O}\left(\alpha_{s}\right)$ terms of the hard coefficient functions $H_{i}$. This programme has recently been completed: the corrections for the topological tree amplitudes have been computed in [2] and the ones for the QCD and electroweak penguin amplitudes in [3].

The work in [4] follows an alternative approach to compute the kernels $T_{i}^{I I}$ (for the tree amplitudes). In this work the calculation has been performed in pure QCD by expanding the 1-loop diagrams to leading power in $1 / m_{b}$. The calculation thus yields directly the convolution from (2.1) without disentangling hard and hard-collinear effects. The findings of [4] agree with those from $[2,9]$, which demonstrates the equivalence of the diagrammatical approach (QCDF) and the effective field theory formulation (SCET) at a non-trivial fixed order in perturbation theory.

\section{Vertex corrections}

The calculation of the kernels $T_{i}^{I}$ is conceptually simpler, since the complicated interplay of soft and collinear dynamics, which induces the hard-collinear degrees of freedom, is absent in this case. The calculation is, however, technically demanding as it amounts to a 2-loop calculation with four external legs, cf. Figure 2.

The 2-loop corrections to the kernels $T_{i}^{I}$ for the topological tree amplitudes have been computed in [5]. The work makes use of a couple of advanced techniques, which are widely applied in multi-loop calculations. First and foremost the calculation is based on an automatized reduction algorithm, which uses integration-by-parts techniques [10] to relate the entire 2-loop calculation to an irreducible set of scalar Master Integrals (MIs). The actual number of 36 MIs turns out to
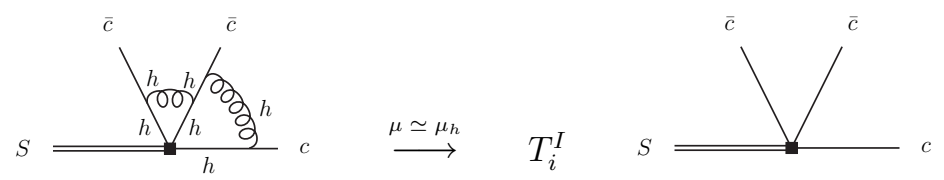

Figure 2: Matching of the 2-loop vertex corrections (the spectator quark is irrelevant for this contribution and not drawn). The different configurations are described in the caption of Figure 1. 
be large and the presence of soft and collinear infrared divergences in intermediate steps of the calculation implies that the MIs are required to up to five coefficients in the $\varepsilon$-expansion, which makes the calculation somewhat involved $(\varepsilon=(4-d) / 2$ in dimensional regularization).

The whole set of MIs has been computed in [5]. Several sophisticated techniques, among these the method of differential equations [11], the formalism of Harmonic Polylogarithms [12] and Mellin-Barnes techniques [13], have been applied to derive analytical expressions for all MIs. The results have further been checked numerically with the method of sector decomposition [14]. About half of these MIs have later been confirmed and applied in a calculation for inclusive semileptonic $B \rightarrow X_{u} \ell v$ decays by various groups [15]. One particular coefficient of one of the most complicated MIs has first been given numerically in [5, 15] and later been rederived in an analytical form [16].

Apart from the calculation of 2-loop integrals, the NNLO calculation of the kernels $T_{i}^{I}$ reveals further technical difficulties. The factorization formula (1.1) implies in particular that the (infrared divergent) contributions, which belong to the formal expansion of the non-perturbative objects $\left(F^{B M}, f_{M}, \phi_{M}\right)$, have to be subtracted from the partonic calculation. In dimensional regularization this matching calculation involves non-physical (evanescent) operators. In order to assure that these operators disappear from the final factorization formula, their renormalized matrix elements have to be made to vanish. The specific pattern of required infrared subtractions is particularly complicated in the calculation of the colour-suppressed tree amplitude, which involves a Fierzevanescent operator already at tree level.

The calculation itself provides a couple of stringent cross-checks. Whereas individual 2-loop diagrams contain up to $1 / \varepsilon^{4}$ (soft and collinear) infrared divergences, the kernels $T_{i}^{I}$ turn out (as predicted) to be free of any singularities, which follows after an intricate subtraction procedure of ultraviolet and infrared divergences. Moreover, somewhat subtle renormalization scheme dependent contributions enter at the 2-loop level scale-dependent terms, which can also be checked. Another important check of the scheme independence of the NNLO results would be possible given a Fierz-symmetric renormalization scheme of evanescent operators. Unfortunately, such a scheme has not yet been worked out at NNLO and therefore this final check is currently lacking.

In view of the complexity of the considered calculation, it is desirable that there are (at least) two independent calculations of the kernels $T_{i}^{I}$ (as for the spectator scattering kernels $T_{i}^{I I}$ ). One ongoing calculation of the tree amplitudes is close to finish [17]. The only missing ingredient of the NNLO calculation then consists in the calculation of the kernels $T_{i}^{I}$ for the penguin amplitudes [18].

\section{Compilation of NNLO results}

\subsection{Conceptual issues}

First of all it is worth noting that factorization has been found in all available NNLO contributions to work technically, i.e. soft and collinear infrared singularities factorize as predicted at NNLO and the resulting convolution integrals are finite (in particular free of endpoint singularities).

Another important conceptual point deals with the question if the dynamical hard-collinear scale $\mu_{h c} \simeq \sqrt{m_{b} \Lambda_{\mathrm{QCD}}}$ should be treated as a perturbative scale, an issue that has been controversial in the literature [7, 19]. The question is related to the perturbative expansion of the spectator scattering mechanism discussed in Section 2. As this contribution starts at $\mathscr{O}\left(\alpha_{s}\right)$, the NNLO terms 
constitute the first radiative corrections to this mechanism. The factorization of this contribution within SCET, as outlined in Section 2, further allows to systematically disentangle the effects from the scales $\mu_{h}$ and $\mu_{h c}$ and in addition to resum logarithms $\ln \mu_{h} / \mu_{h c}$ via renormalization group techniques. The explicit NNLO results [2, 3, 4, 9] do not show any sign of abnormally large corrections and the remnant dependence on the scales $\mu_{h}$ and $\mu_{h c}$ turns out to be well-behaved. The NNLO calculation thus suggests that the expansion in $\alpha_{s}\left(\mu_{h c}\right)$ is under control.

\subsection{Tree amplitudes}

One important subset of hard-scattering kernels, which specify the topological tree amplitudes, are by now completely determined to NNLO $[2,4,5]$. It is interesting to illustrate the structure of the perturbative expansion at the amplitude level. Using the input parameters specified in [20], the colour-allowed $\left(\alpha_{1}\right)$ and colour-suppressed $\left(\alpha_{2}\right)$ tree amplitudes in the $B \rightarrow \pi \pi$ channels become

$$
\begin{aligned}
\alpha_{1}(\pi \pi)= & \left.1.008\right|_{V^{(0)}}+[0.025+0.010 i]_{V^{(1)}}+[0.027+0.032 i]_{V^{(2)}} \\
& -\left.0.012\right|_{S^{(1)}}-[0.021+0.015 i]_{S^{(2)}}-\left.0.014\right|_{P} \\
= & 1.013_{-0.036}^{+0.023}+\left(+0.027_{-0.022}^{+0.025}\right) i \\
\alpha_{2}(\pi \pi)= & \left.0.223\right|_{V^{(0)}}-[0.174+0.075 i]_{V^{(1)}}-[0.032+0.051 i]_{V^{(2)}} \\
& +\left.0.090\right|_{S^{(1)}}+[0.034+0.025 i]_{S^{(2)}}+\left.0.055\right|_{P} \\
= & 0.195_{-0.089}^{+0.134}+\left(-0.101_{-0.063}^{+0.061}\right) i
\end{aligned}
$$

where the various terms of the perturbative expansion have been denoted by $V^{(0)}$ corresponding to the tree level contribution, $V^{(1)}$ (1-loop vertex corrections) and $S^{(1)}$ (tree level spectator scattering) to the NLO corrections and $V^{(2)}$ (2-loop vertex corrections) and $S^{(2)}$ (1-loop spectator scattering) to the new NNLO terms, whereas the numbers denoted by $P$ give an estimate of power corrections $\sim 1 / m_{b}$ that are related to subleading twist wave functions of the pions.

From (4.1) it is obvious that the NNLO corrections are particularly important for the imaginary parts of the amplitudes and hence for strong phases and direct CP asymmetries, which are first generated at $\mathscr{O}\left(\alpha_{s}\right)$. The new corrections are in some cases found to exceed the NLO terms, which can be explained by a numerical enhancement from the Wilson coefficients. In absolute terms, however, the new corrections are small and perturbation theory seems to be well-behaved.

The uncertainties of the NNLO prediction for the colour-allowed tree amplitude $\alpha_{1}$ are at a satisfactory level of few percent. The colour-suppressed amplitude $\alpha_{2}$, on the other hand, suffers from substantial uncertainties, which can be traced back to the strong cancellation between the terms denoted by $V^{(0)}, V^{(1)}$ and $V^{(2)}$. This makes the real part of $\alpha_{2}$ particularly sensitive to the spectator scattering mechanism, which is proportional to the hadronic ratio $f_{\pi} \hat{f}_{B} / \lambda_{B} F_{+}^{B \pi}(0)$. The poor knowledge of the $B$ meson parameter $1 / \lambda_{B} \equiv \int_{0}^{\infty} d \omega / \omega \phi_{B}(\omega)$ in particular makes the theoretical prediction of $\alpha_{2}$ rather uncertain, which calls for further theoretical progress from nonperturbative methods (the numbers are given for $\left.\lambda_{B}=(400 \pm 150) \mathrm{MeV}\right)$.

\subsection{The approximate tree decays $B^{-} \rightarrow \pi^{-} \pi^{0} / \rho^{-} \rho^{0}$}

Isospin symmetry implies that the decays $B^{-} \rightarrow \pi^{-} \pi^{0} / \rho^{-} \rho^{0}$ are free of QCD penguin contributions (they depend, however, on small electroweak penguin amplitudes). As they do not re- 
ceive contributions from weak annihilation either, which constitutes an important class of nonfactorizable power corrections to the factorization formula (1.1), their branching ratios are particularly suited to test the strong interaction dynamics of the tree amplitudes. Treating the electroweak penguin amplitudes in the NLO approximation [1, 21], the QCDF prediction becomes [20]

$$
\begin{aligned}
& 10^{6} \operatorname{Br}\left(B^{-} \rightarrow \pi^{-} \pi^{0}\right)=6.22_{-1.05-1.65-0.18-0.42}^{+1.14+2.03+0.16+0.43} \\
& 10^{6} \operatorname{Br}\left(B^{-} \rightarrow \rho_{L}^{-} \rho_{L}^{0}\right)=21.0_{-3.5-6.5_{-0.7-1.4}^{+3.9+7.4+0.5}}^{+1.5}
\end{aligned}
$$

which is in good agreement with the experimental values given in parentheses. Here $L$ refers to the longitudinal polarization and the uncertainties are, in order, due to CKM parameters, hadronic parameters, higher order perturbative corrections and power corrections. The theoretical prediction depends, however, strongly on the input values for $\left|V_{u b}\right|$ and the form factors $F_{+}^{B \pi}(0)$ and $A_{0}^{B \rho}(0)$.

The theoretical prediction can be made independent of these input parameters by normalizing the hadronic decay rates to the differential semileptonic decay rates at maximum recoil. The ratio

$$
\frac{\Gamma\left(B^{-} \rightarrow \pi^{-} \pi^{0}\right)}{d \Gamma\left(\bar{B}^{0} \rightarrow \pi^{+} \ell^{-} \bar{v}_{l}\right) /\left.d q^{2}\right|_{q^{2}=0}} \simeq 3 \pi^{2} f_{\pi}^{2}\left|V_{u d}\right|^{2}\left|\alpha_{1}(\pi \pi)+\alpha_{2}(\pi \pi)\right|^{2}
$$

provides an exceptionally clean probe of the QCD dynamics of the tree amplitudes [22]. The NNLO prediction for this ratio $\left(0.70_{-0.08}^{+0.12}\right) \mathrm{GeV}^{2}$ compares again well to the experimental value $\left(0.81_{-0.14}^{+0.14}\right) \mathrm{GeV}^{2}$, which strongly supports the factorization assumption [20]. It would, however, be interesting to see if the tendency between the experimental and the theoretical value is reproduced in the $\rho$-sector. As the semileptonic $B \rightarrow \rho \ell v$ decay spectrum has not yet been measured precisely, one may instead consider a ratio of two hadronic decay rates

$$
\frac{\Gamma\left(B^{-} \rightarrow \rho_{L}^{-} \rho_{L}^{0}\right)}{\Gamma\left(\bar{B}^{0} \rightarrow \rho_{L}^{+} \rho_{L}^{-}\right)} \simeq \frac{\left|\alpha_{1}\left(\rho_{L} \rho_{L}\right)+\alpha_{2}\left(\rho_{L} \rho_{L}\right)\right|^{2}}{2\left|\alpha_{1}\left(\rho_{L} \rho_{L}\right)\right|^{2}}
$$

which, in contrast to (4.3), receives corrections from QCD penguin amplitudes and weak annihilation. The NNLO prediction for this ratio $\left(0.65_{-0.11}^{+0.16}\right)$ is again found to be somewhat smaller than the experimental value $\left(0.89_{-0.14}^{+0.14}\right)$, which may be considered as a hint at somewhat enhanced colour-suppressed amplitudes that could be realized in QCDF by a smaller value of the $B$ meson parameter $\lambda_{B} \simeq 250 \mathrm{MeV}[20]$.

\section{Conclusions}

The NNLO calculation for charmless hadronic $B$ meson decays is particularly important in respect of direct $\mathrm{CP}$ asymmetries that are first generated at $\mathscr{O}\left(\alpha_{s}\right)$. It opens in particular a new mechanism from spectator scattering that induces strong phases and settles some conceptual aspects that bring the factorization framework onto a more rigorous footing.

Whereas the topological tree amplitudes are by now completely determined to NNLO, the computation of the penguin amplitudes is to date still incomplete. Further improvements on the calculation require a better understanding of power corrections, in particular on the role of the phenomenologically important scalar penguin amplitude, and more precise determinations of hadronic input parameters. 


\section{References}

[1] M. Beneke, G. Buchalla, M. Neubert and C. T. Sachrajda, Phys. Rev. Lett. 83 (1999) 1914, Nucl. Phys. B 591 (2000) 313, Nucl. Phys. B 606 (2001) 245.

[2] M. Beneke and S. Jäger, Nucl. Phys. B 751 (2006) 160; N. Kivel, JHEP 0705 (2007) 019.

[3] M. Beneke and S. Jäger, Nucl. Phys. B 768 (2007) 51; A. Jain, I. Z. Rothstein and I. W. Stewart, arXiv:0706.3399 [hep-ph].

[4] V. Pilipp, PhD thesis, LMU München, 2007, arXiv:0709.0497 [hep-ph]; Nucl. Phys. B 794 (2008) 154.

[5] G. Bell, Nucl. Phys. B 795 (2008) 1; PhD thesis, LMU München, 2006, arXiv:0705.3133 [hep-ph]; arXiv:0902.1915 [hep-ph], to be published in Nucl. Phys. B.

[6] C. W. Bauer, S. Fleming, D. Pirjol and I. W. Stewart, Phys. Rev. D 63 (2001) 114020; C. W. Bauer, D. Pirjol and I. W. Stewart, Phys. Rev. D 65 (2002) 054022; M. Beneke, A. P. Chapovsky, M. Diehl and T. Feldmann, Nucl. Phys. B 643 (2002) 431.

[7] C. W. Bauer, D. Pirjol, I. Z. Rothstein and I. W. Stewart, Phys. Rev. D 70 (2004) 054015.

[8] M. Beneke and T. Feldmann, Nucl. Phys. B 592 (2001) 3; C. W. Bauer, D. Pirjol and I. W. Stewart, Phys. Rev. D 67 (2003) 071502; M. Beneke and T. Feldmann, Nucl. Phys. B 685 (2004) 249; B. O. Lange and M. Neubert, Nucl. Phys. B 690 (2004) 249 [Erratum-ibid. B 723 (2005) 201].

[9] R. J. Hill, T. Becher, S. J. Lee and M. Neubert, JHEP 0407 (2004) 081; T. Becher and R. J. Hill, JHEP 0410 (2004) 055; G. G. Kirilin, arXiv:hep-ph/0508235; M. Beneke and D. Yang, Nucl. Phys. B 736 (2006) 34.

[10] F. V. Tkachov, Phys. Lett. B 100 (1981) 65; K. G. Chetyrkin and F. V. Tkachov, Nucl. Phys. B 192 (1981) 159.

[11] A. V. Kotikov, Phys. Lett. B 254 (1991) 158; E. Remiddi, Nuovo Cim. A 110 (1997) 1435.

[12] E. Remiddi and J. A. M. Vermaseren, Int. J. Mod. Phys. A 15 (2000) 725.

[13] V. A. Smirnov, Phys. Lett. B 460 (1999) 397; J. B. Tausk, Phys. Lett. B 469 (1999) 225.

[14] T. Binoth and G. Heinrich, Nucl. Phys. B 585 (2000) 741.

[15] R. Bonciani and A. Ferroglia, JHEP 0811 (2008) 065; H. M. Asatrian, C. Greub and B. D. Pecjak, Phys. Rev. D 78 (2008) 114028; M. Beneke, T. Huber and X. Q. Li, Nucl. Phys. B 811 (2009) 77; G. Bell, Nucl. Phys. B 812 (2009) 264.

[16] T. Huber, JHEP 0903 (2009) 024.

[17] T. Huber, talk at DPG meeting, München, 2009; M. Beneke, T. Huber and X. Q. Li, in preparation.

[18] G. Bell, M. Beneke, T. Huber and X. Q. Li, in preparation.

[19] M. Beneke, G. Buchalla, M. Neubert and C. T. Sachrajda, Phys. Rev. D 72 (2005) 098501; C. W. Bauer, D. Pirjol, I. Z. Rothstein and I. W. Stewart, Phys. Rev. D 72 (2005) 098502.

[20] G. Bell and V. Pilipp, arXiv:0907.1016 [hep-ph].

[21] M. Beneke, J. Rohrer and D. Yang, Nucl. Phys. B 774 (2007) 64; M. Bartsch, G. Buchalla and C. Kraus, arXiv:0810.0249 [hep-ph].

[22] J. D. Bjorken, Nucl. Phys. Proc. Suppl. 11 (1989) 325; M. Beneke and M. Neubert, Nucl. Phys. B 675 (2003) 333. 